\title{
Magnetic force microscope tip-induced remagnetization of CoPt nanodisks with perpendicular anisotropy
}

\author{
V. L. Mironov, ${ }^{1, a)}$ B. A. Gribkov, ${ }^{1}$ S. N. Vdovichev, ${ }^{1}$ S. A. Gusev, ${ }^{1}$ A. A. Fraerman, ${ }^{1}$ \\ O. L. Ermolaeva, ${ }^{1}$ A. B. Shubin, ${ }^{2}$ A. M. Alexeev, ${ }^{2}$ P. A. Zhdan, ${ }^{3}$ and C. Binns ${ }^{4}$ \\ ${ }_{1}^{1}$ Institute for Physics of Microstructures, RAS, Nizhniy Novgorod 603950, Russia \\ 2"Nanotechnology MDT" Company, Zelenograd 124482, Russia \\ ${ }^{3}$ School of Engineering, University of Surrey, Guildford, Surrey GU2 7XH, United Kingdom \\ ${ }^{4}$ Department of Physics and Astronomy, University of Leicester, Leicester LE1 7RH, United Kingdom
}

(Received 18 May 2009; accepted 14 July 2009; published online 14 September 2009)

\begin{abstract}
We report on the results of a magnetic force microscopy investigation of remagnetization processes in arrays of CoPt nanodisks with diameters of 35 and $200 \mathrm{~nm}$ and a thickness of $9.8 \mathrm{~nm}$ fabricated by e-beam lithography and ion etching. The controllable magnetization reversal of individual CoPt nanodisks by the magnetic force microscope (MFM) tip-induced magnetic field was demonstrated. We observed experimentally two essentially different processes of tip-induced remagnetization. Magnetization reversal of $200 \mathrm{~nm}$ disks was observed when the probe moved across the particle while in case of $35 \mathrm{~nm}$ nanodisks one-touch remagnetization was realized. Micromagnetic modeling based on the Landau-Lifshitz-Gilbert (LLG) equation demonstrated that the tip-induced magnetization reversal occurs through the essentially inhomogeneous states. Computer simulations confirmed that in case of $200 \mathrm{~nm}$ disks the mechanism of embryo nucleation with reversed magnetization and further dynamic propagation following the probe moving across the particle was realized. On the other hand one-touch remagnetization of $35 \mathrm{~nm}$ disks occurs through the inhomogeneous vortexlike state. Micromagnetic LLG simulations showed that magnetization reversal in an inhomogeneous MFM probe field has a lower energy barrier in comparison with the mechanism of coherent rotation, which takes place in a homogeneous external magnetic field. (C) 2009 American Institute of Physics. [doi:10.1063/1.3202354]
\end{abstract}

\section{INTRODUCTION}

Bit-patterned recording media and especially arrays of dots with perpendicular magnetic anisotropy are very promising for the realization of data storage densities up to $1 \mathrm{Tbit} / \mathrm{in.}^{2}{ }^{1-7}$ In this connection considerable efforts have been applied to the investigation of coercivity, thermal stability, and recording properties of patterned systems. ${ }^{8-16}$ However most studies focused on the investigation of the array's averaged properties and inhomogeneous dot's switching in an external homogeneous magnetic field. In this article we discuss the peculiarities of the magnetization reversal of individual dots in a nonhomogeneous magnetic field. The simplest way to realize a highly inhomogeneous magnetic field is to use a magnetic force microscope (MFM) tip. In this case, one can change the magnetic state of the particle and simultaneously analyze the new state. The influence of the MFM probe on the magnetic states of ferromagnetic nanoparticles was partly discussed in Refs. 17-19, but the efforts were concentrated on the study of the in-plane magnetization reversal of uniform magnetized single domain elliptical nanodisks. Previously we also reported the influence of the MFM probe inhomogeneous magnetic field on the in-plane remagnetization processes in elliptical ferromagnetic nanodisks. ${ }^{20-22}$ We showed experimentally and by micromagnetic simulations that nonsymmetrical scanning of the inhomogenous tip field over the particle allows one to

${ }^{a)}$ Electronic mail: mironov@ipm.sci-nnov.ru. induce transitions between uniform states with different orientations and between uniform and vortex states. In the present paper we report on the processes involved in tipinduced remagnetization in circular CoPt nanodisks with perpendicular magnetic anisotropy. We demonstrate the possibility of controllable reading/writing and discuss the mechanisms of tip-induced magnetization reversal and its dependence on tip and particle sizes.

\section{SAMPLE PREPARATION AND EXPERIMENTAL TECHNIQUE}

The initial CoPt multilayer thin film consisting of seven bilayers of $\mathrm{Co}(4 \AA) / \mathrm{Pt}(10 \AA)$ was grown by magnetron sputtering on silicon substrates at the University of Nebraska-Lincoln. ${ }^{23,24}$ The film was protected by a $20 \mathrm{~nm}$ thick $\mathrm{SiO}_{2}$ overcoat. This multilayer structure demonstrated perpendicular magnetic anisotropy and had a square hysteresis loop with a coercitivity of 200 Oe. The two arrays of nanoparticles in the form of circular disks with diameters of 35 and $200 \mathrm{~nm}$ were fabricated by electron beam lithography and ion beam etching. The spatial periods of these arrays were 120 and $500 \mathrm{~nm}$, respectively. Some details of the lithographic processes are described in Ref. 25.

Scanning electron microscopy (SEM) investigations and e-beam lithography were performed using a "SUPRA 50VP" electron microscope ("Carl Zeiss" Co.). The MFM investigations of the magnetic states and remagnetization effects in $\mathrm{CoPt}$ nanoparticles were carried out using a vacuum scan- 
ning probe microscope "Solver HV" ("NT-MDT" Co.). MFM measurements were performed in the oscillatory noncontact (constant height) mode using home made MFM cantilevers with Co coating with $20-50 \mathrm{~nm}$ thickness. In imaging mode the double-amplitude of the cantilever oscillations was about $30 \mathrm{~nm}$ and the average scanning height was 50 $\mathrm{nm}$. The phase shift of the cantilever oscillations caused by the gradient of the sample magnetic field was used to obtain MFM contrast. All measurements were performed in a vacuum of $10^{-3}$ Torr, which increases the MFM signal relative to an in-air measurement due to an increase in the cantilever quality factor.

\section{COMPUTER SIMULATIONS}

Modeling of remagnetization processes in nanodisks under an MFM probe magnetic field was performed using specially developed software based on solving the LandauLifshitz-Gilbert (LLG) equation for the magnetization $\vec{M}(\vec{r}, t)$ :

$$
\frac{\partial \vec{M}}{\partial t}=-\frac{\gamma}{1+\alpha^{2}}\left[\vec{M} \vec{H}_{\mathrm{eff}}\right]-\frac{\alpha \gamma}{\left(1+\alpha^{2}\right) M_{s}}\left[\vec{M}\left[\vec{M} \vec{H}_{\mathrm{eff}}\right]\right],
$$

where $\gamma$ is the gyromagnetic ratio, $\alpha$ is the dimensionless damping parameter, and $M_{S}$ is the magnetic moment at saturation. The effective field $\vec{H}_{\text {eff }}=-\delta E / \delta \vec{M}$ is a variation derivative of the energy function. The total energy of the particle can be defined by

$$
E=E_{e x}+E_{m}+E_{a}+E_{h} .
$$

The first term $E_{e x}$ is the energy of the exchange interaction, the second term $E_{m}$ is the demagnetization energy of the disk, and $E_{a}$ is the magnetic anisotropy energy. Expressions for these terms have conventional form. ${ }^{26}$ The last term $E_{h}$ is the energy of the interaction between the particle magnetization and an external magnetic field $\vec{H}$. An external uniform magnetic field and the magnetic field of an MFM probe were applied as $\vec{H}$ in computer simulations. Both the dynamics of the magnetization in a CoPt nanodisk $\left[M_{x}(\vec{r}, t), M_{y}(\vec{r}, t), M_{z}(\vec{r}, t)\right]$ and the averaged total energy of the disk $\bar{E}$ were calculated as a function of the magnetic field $\vec{H}$.

In the calculations the MFM tip was approximated by a uniformly magnetized sphere with an effective magnetic moment, $m_{t}=M_{t} V_{t}$, where $M_{t}$ is the remanent magnetization of the tip capping material and $V_{t}$ is the effective volume of the interactive part of the tip. Analysis of experimental data shows that the typical effective volume of magnetic material for our probes with a Co coating $\left(M_{t} \sim 1400 \mathrm{G}\right)$ is approximately $1 \times 10^{5}-5 \times 10^{5} \mathrm{~nm}^{3}$, that is, the radius of the effective sphere $R_{t}$ is $30-50 \mathrm{~nm}$.

We studied the dynamics of the magnetization in an external uniform field as well as the redistribution of the magnetization during the motion of the probe across the particle and perpendicular to it right down to contact. In the case of a moving MFM tip, for each position, the stationary solutions of the LLG equations for the particle magnetization in the inhomogeneous field of the MFM tip were found. After find-
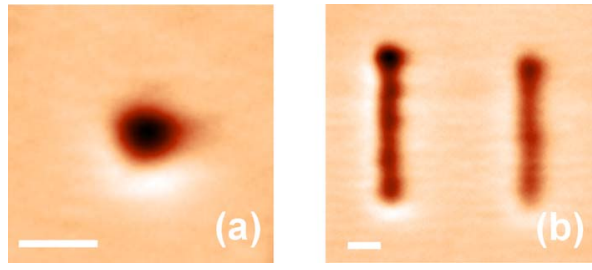

FIG. 1. (Color online) The MFM tip produced domains with reversed magnetization in a CoPt thin film with perpendicular magnetic anisotropy. (a) is the MFM image of a circular domain obtained by one-touch inversion. (b) is the MFM image of linear domains obtained by selective scanning in contact mode. (The white scale bars are $1 \mu \mathrm{m}$.)

ing the solution at a given position the probe was moved and the LLG equations were solved again with the previous magnetization distribution used as the initial state for the next step computation. The reverse influence of sample magnetization on the tip magnetic moment was neglected since in our experiments the coercivity of MFM tip was larger than that of the CoPt nanodisks.

The calculations were carried out for the following CoPt disks parameters: the constant of exchange interaction was $J=10^{-6} \mathrm{erg} / \mathrm{cm}$, the remagnetization in saturation was $M_{s}$ $=800 \mathrm{emu} / \mathrm{cm}^{3}$, and the anisotropy constant, $K$, was in the range from $5 \times 10^{6}$ to $5 \times 10^{7} \mathrm{erg} / \mathrm{cm}^{3}{ }^{27-30}$

\section{EXPERIMENTAL RESULTS AND DISCUSSION}

The preliminary experiments on the tip remagnetization of CoPt films showed that the characteristic size of a stable domain with reversed magnetization was about $500 \mathrm{~nm}$. As an example, the MFM images of a CoPt film with circular and strip shape domains of reversed magnetization are represented in Fig. 1. The film was initially magnetized in the opposite direction to the MFM probe magnetic moment. The reversed domains were formed by the influence of the magnetic tip in contact mode.

For the tip-induced remagnetization experiments on patterned films, we fabricated arrays of nanodisks with diameters smaller than the stable domain dimension. As we show below, we expect the tip-induced remagnetization process to be different for the two sizes of nanodisk. We considered the simple but realistic model of uniform magnetized parabolic MFM probe with magnetic coating (Fig. 2).

The $h$ and $\rho(\rho=|\vec{\rho}|)$ components of the magnetic field of the parabolic probe can be represented by the following expressions:

$$
\begin{aligned}
& H_{h}(\rho, h)=M_{t} \int_{V_{t}} \frac{2(z+h)^{2}-(\vec{\rho}-\vec{r})^{2}}{\left[(h+z)^{2}+(\vec{\rho}-\vec{r})^{2}\right]^{5 / 2}} d V, \\
& H_{\rho}(\rho, h)=M_{t} \int_{V_{t}} \frac{3(z+h)|\vec{\rho}-\vec{r}|}{\left[(h+z)^{2}+(\vec{\rho}-\vec{r})^{2}\right]^{5 / 2}} d V .
\end{aligned}
$$

For example, the results of numerical calculations for the endless parabolic MFM probe with the parameters $a=0.025$ (parabolic coefficient) and $d=30 \mathrm{~nm}$ (magnetic coating thickness), which are representative of experimental values, are shown in Fig. 3. We introduced the normalized values $\tilde{H}_{h}=H_{h} / H_{h}(0, \delta), \tilde{H}_{\rho}=H_{\rho} / H_{z}(0, \delta)$, and $\widetilde{\rho}=\rho / R_{t}$, where the 


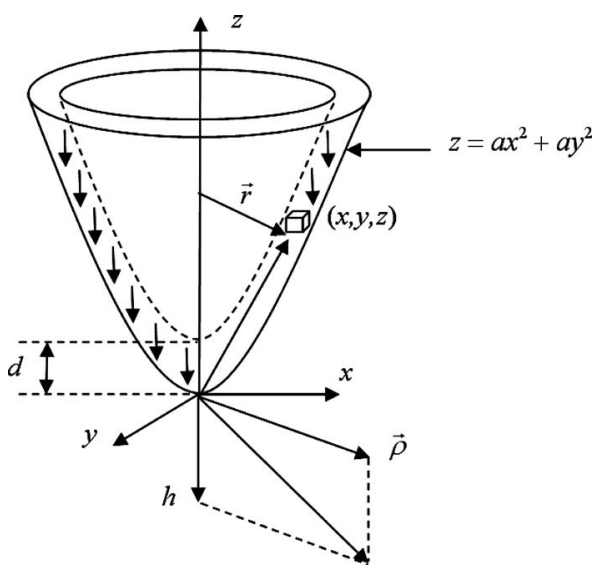

FIG. 2. The parabolic model of the MFM probe with magnetic coating. The $x, y$, and $z$ are the coordinates of integration $\left(r^{2}=x^{2}+y^{2}\right)$, while $h$ and $\vec{\rho}$ are the coordinates of the observation point

disk's capping layer thickness $\delta=20 \mathrm{~nm}$ and the spherical probe radius $R_{t}=30 \mathrm{~nm}$. The $h$ component of the magnetic stray field near the surface of the parabolic probe $(h=0, \rho$ $=0)$ can be written as

$$
H_{h}(0,0)=\frac{16 \pi a d M_{t}}{(1+4 a d)} .
$$

This value for Co based probes $(a=0.025, d=30 \mathrm{~nm})$ is about $13 \mathrm{kOe}$ and exceeds the coercivity of CoPt nanodisks.

On the other hand the MFM probe can be approximated by the effective spherical probe. In the case of the spherical probe, we have more simple expressions for the $h$ and $\rho$ components of probe's field

$$
\begin{aligned}
& H_{h}(\rho, h)=\frac{4 \pi M_{t} R_{t}^{3}}{3} \frac{2\left(h+R_{t}\right)^{2}-\rho^{2}}{\left[\left(h+R_{t}\right)^{2}+\rho^{2}\right]^{5 / 2}}, \\
& H_{\rho}(\rho, h)=4 \pi M_{t} R_{t}^{3} \frac{\left(h+R_{t}\right) \rho}{\left[\left(h+R_{t}\right)^{2}+\rho^{2}\right]^{5 / 2}} .
\end{aligned}
$$

The spatial distributions of $h$ and $\rho$ components of the magnetic fields (6) and (7) for the spherical probe with $R_{t}$ $=30 \mathrm{~nm}$ are represented in Figs. 3(a) and 3(b). It is seen that expressions (6) and (7) give us the quite reliable approximation of the parabolic probe's field but they are more appropriate for LLG simulations.
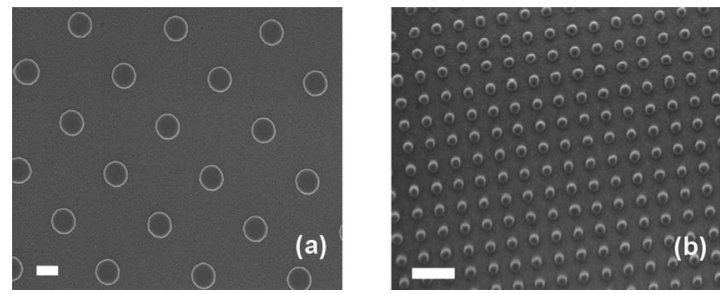

FIG. 4. SEM images of CoPt nanodisks arrays. (a) is for the $200 \mathrm{~nm}$ nanodisk array with a period of $500 \mathrm{~nm}$. (b) is for the $35 \mathrm{~nm}$ nanodisk array with a period of $120 \mathrm{~nm}$. (The white scale bars are $200 \mathrm{~nm}$.)

Moreover it seen from Fig. 3 that the characteristic spatial scale of the magnetic field is comparable with the probe radius $\rho \sim R_{t}$. Therefore the remagnetization process should differ for the CoPt disks with $R_{d}>R_{t}$ and for $R_{d}<R_{t}$.

We fabricated two arrays of CoPt circular nanodisks with diameter $D_{d}=200 \mathrm{~nm}\left(R_{d}>R_{t}\right)$ and $D_{d}=35 \mathrm{~nm}\left(R_{d}\right.$ $<R_{t}$ ). The typical SEM images (plan view in secondary electrons) of fabricated CoPt nanodisks are represented in Fig. 4.

The SEM contrast in this mode (Fig. 4) highlights the disk's edges, ${ }^{31}$ which allows one to control the shape and sizes of particles. To exclude interparticle interaction we fabricated particles arrays with a period $L$ larger than disk's diameter $D_{d}\left(L>2 D_{d}\right)$ and larger than probe diameter $D_{t}$ $\left(L>D_{t}\right)$. Thus the $200 \mathrm{~nm}$ CoPt nanodisks were arranged in a square array with a $500 \mathrm{~nm}$ period and the $35 \mathrm{~nm}$ nanodisks were arranged in a square array with a $120 \mathrm{~nm}$ period.

\section{A. MFM probe induced remagnetization of $200 \mathrm{~nm}$ CoPt nanodisks}

The magnetization reversal of magnetic nanoparticles in an external homogeneous magnetic field has been widely discussed. In general two simple and realistic mechanisms of remagnetization are commonly considered. The first is the Stoner-Wolhlfarth mechanism suggesting that remagnetization occurs through coherent magnetization rotation ${ }^{32-34}$ and the second one is the reversed embryo nucleation and growth. ${ }^{34-37}$ However we expected that remagnetization in the highly inhomogeneous MFM probe field could occur by essentially different processes.

In the first series of experiments we studied the tipinduced remagnetization of $200 \mathrm{~nm}$ CoPt nanodisks $\left(R_{d}\right.$ $\left.>R_{t}\right)$. The sample was previously magnetized in a perpen-
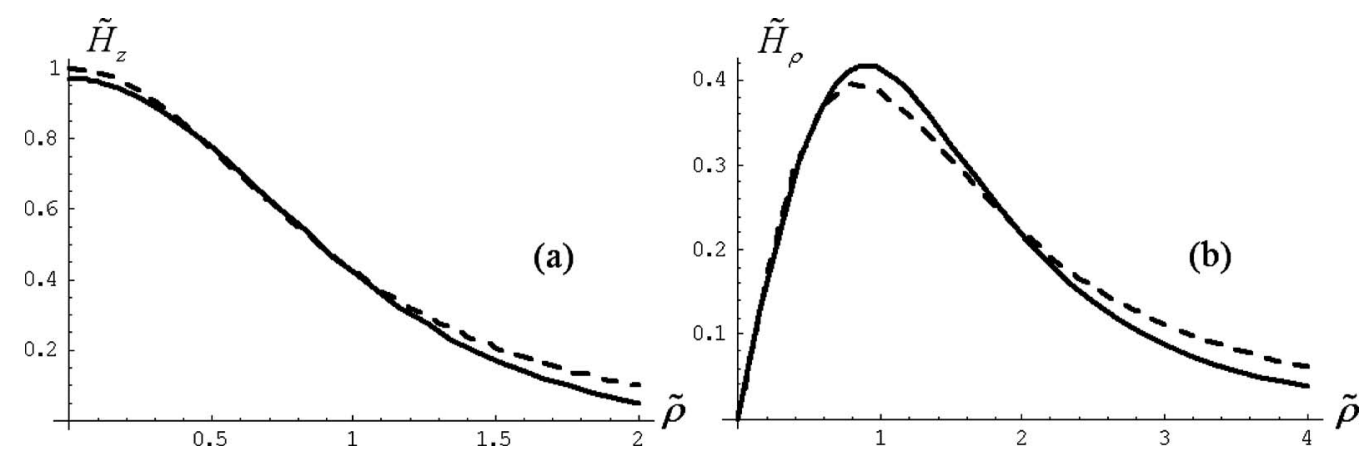

FIG. 3. (a) is the spatial distributions of $\widetilde{H}_{z}(\widetilde{\rho})$ in the plane $h=\delta$ for parabolic (dashed line) and for spherical (solid line) probes. (b) is the spatial distributions of $\widetilde{H}_{\rho}(\widetilde{\rho})$ in the plane $h=\delta$ for parabolic (dashed line) and for spherical (solid line) probes. 


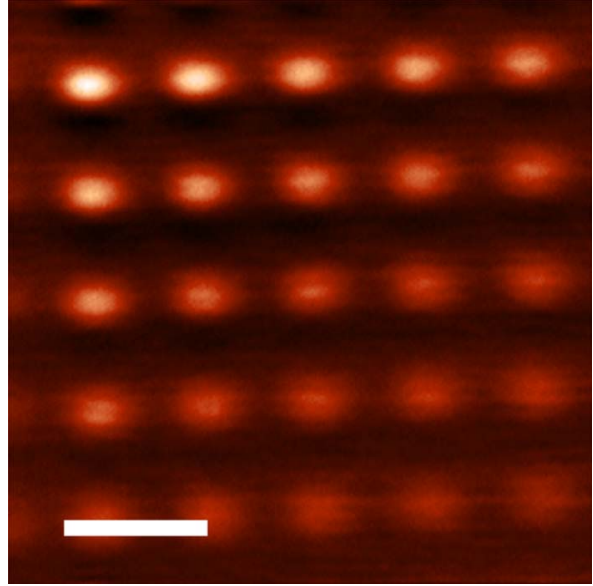

FIG. 5. (Color online) MFM image of the $200 \mathrm{~nm}$ CoPt nanodisk array. The sample has been previously magnetized in the perpendicular $(z)$ direction opposite to the MFM tip moment. (The white scale bar is $1 \mu \mathrm{m}$.)

dicular external field of $15 \mathrm{kOe}$ in the opposite direction to the probe moment. The MFM image of the initial state of CoPt disks array is shown in Fig. 5. It is evident that all particles are in a single-domain state and bright contrast in the MFM image corresponds to the disks areas.

The MFM tip-induced controllable writing on this particle array was investigated experimentally. It was found that a reliable inversion of the particle magnetization was not possible with a one-touch method so we used a different approach. The writing to selected particles was realized by reducing the tip-sample separation during scanning across the particle right up to switching of the magnetization. The step by step MFM images of the controllable writing on the $200 \mathrm{~nm}$ CoPt disks array are shown in Fig. 6 and the detailed images of remagnetization process are shown in Fig. 7.

The images in Fig. 7 demonstrate the remagnetization of an individual $200 \mathrm{~nm}$ CoPt nanodisk by moving the MFM probe across it. The characteristic moment of remagnetization for the selected nanodisk is shown in Fig. 7(b). The probe-particle separation was decreased during probe scanning across central part of disk (at the line $\mathrm{AB}[$ Fig. $7(\mathrm{~b})]$ )
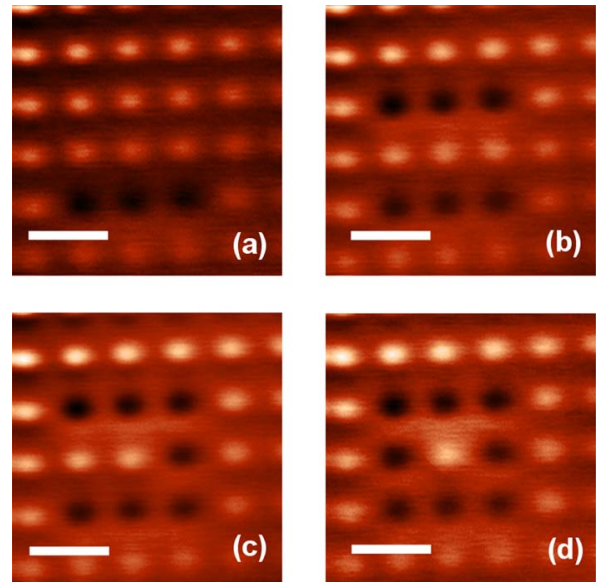

FIG. 6. (Color online) The MFM tip-induced writing in the ordered array of $200 \mathrm{~nm}$ CoPt nanoparticles. (a) is the MFM image with three reversed particles. (b) is for six reversed particles, (c) is for seven reversed particles, (d) is for eight reversed particles. (The white scale bars are $1 \mu \mathrm{m}$.)
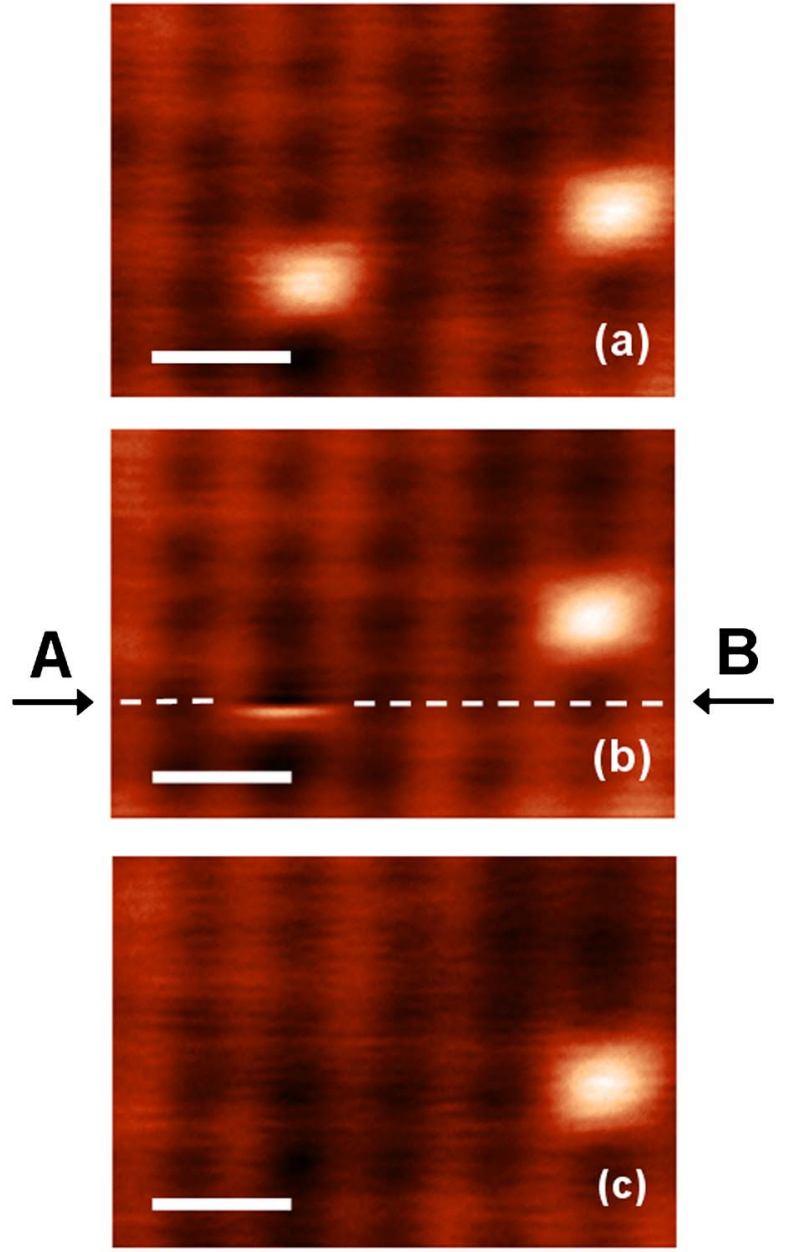

FIG. 7. (Color online) The remagnetization of an individual CoPt nanodisk by moving the MFM probe across it. (a) is the constant height MFM image of the initial state of the CoPt array area with two particles not inverted. (b) is the MFM image obtained in writing mode with a low scanning height along the line AB. The contrast inversion is clearly seen. (c) is the final state of the same area after MFM writing. (The white scale bars are $1 \mu \mathrm{m}$.)

and after sharp MFM contrast inversion was reset again. The final MFM image [Fig. 7(c)] confirms that local remagnetization really took place in this process.

The above mentioned behavior of nanodisks with $R_{d}$ $>R_{t}$ was confirmed in micromagnetic LLG modeling. It was shown that when the spherical MFM probe (radius $50 \mathrm{~nm}$ ) was placed over the center of particle then the local reversed embryo nucleation was observed but the probe field was not large enough for one-touch remagnetization. The results of LLG calculations of the probe induced remagnetization process for $200 \mathrm{~nm}$ CoPt disks are represented in Fig. 8. At the first stage [Fig. 8(b)] the formation of a radial component of the magnetization caused by the radial component of the MFM tip field was observed. After that the nucleation of the reversed embryo through the quasivortex state [Figs. $8(a)-8(c)$ ] was observed. The embryo expands and is stabilized at a lateral diameter of about $150 \mathrm{~nm}$. When the probe is moved out, the relaxation of magnetization to the initial uniform state is observed [Figs. 8(e) and 8(f)]. We ascribe the stability of the $200 \mathrm{~nm}$ particles to edge effects since the remagnetization through domain wall propagation is accompanied by a significant increase in magnetostatic energy at 


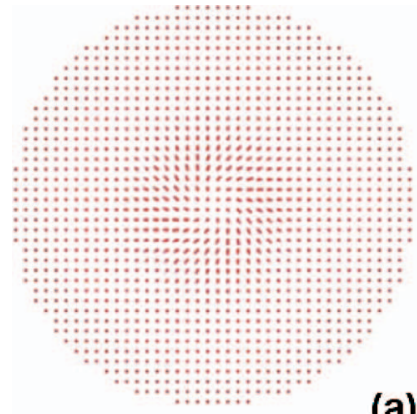

(a)
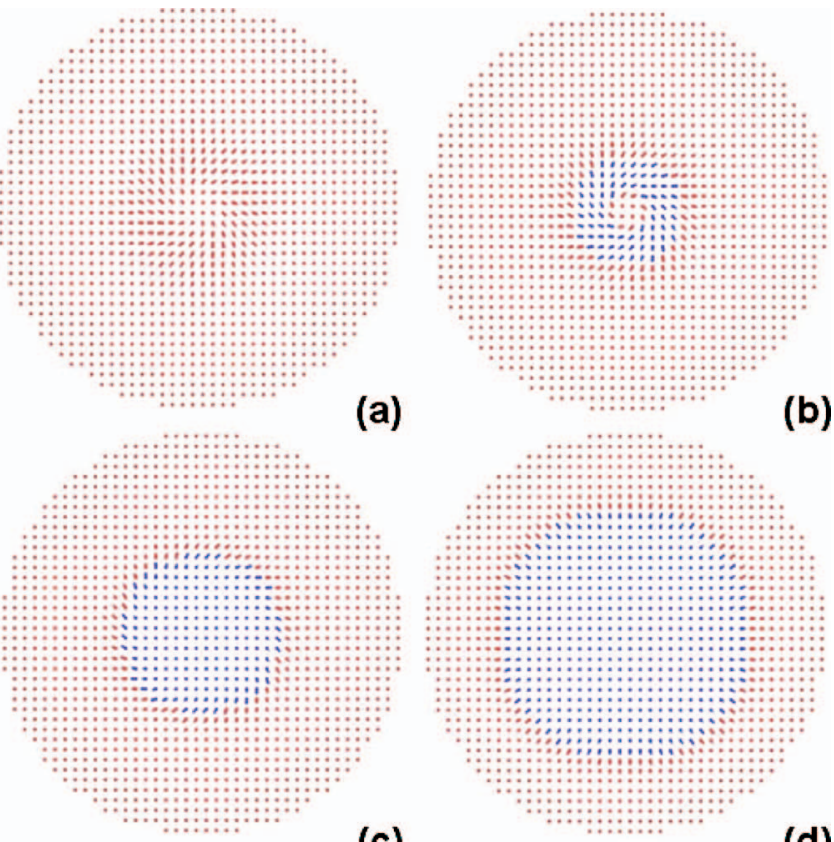

(c)
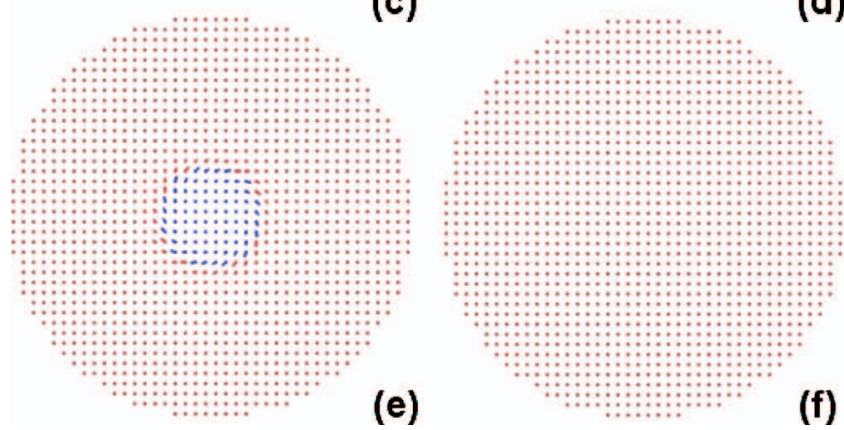

FIG. 8. (Color) Step-by-step stages of the remagnetization process for 200 $\mathrm{nm}$ CoPt disk under the MFM probe magnetic field (at a temperature of 0 $\mathrm{K})$. The spherical $\left(R_{t}=50 \mathrm{~nm}\right)$ probe is situated over the center of particle. (a)-(d) are magnetization distributions at different times during the remagnetization process. The stable state corresponds to picture (d). When the probe was moved out the relaxation of the magnetization to the initial state is observed [(e) and (f)]. The positive $Z$-component of the magnetization is indicated by red and the negative $Z$-component by blue.

the disk's edge. The increase in coercivity of particles in comparison with the coercivity of a continuous film was also discussed in Refs. 34 and 10.

The simulations were also performed for remagnetization by MFM probe scanning. The step by step model pictures of the remagnetization process during probe scanning across the $200 \mathrm{~nm}$ CoPt nanodisk are represented in Fig. 9. The initial state [Fig. 9(a)] corresponds to the uniform magnetization directed up along the $Z$ axis (indicated by the red color). At the first stage the spherical MFM probe (radius 50 $\mathrm{nm}$ ) was placed over the edge of the nanodisk [Fig. 9(b)] and part of magnetization was reversed under the field of the tip. In fact the strong local probe field overcomes the barrier for remagnetization caused by the particle edge. Further as the tip moves across the particle it is followed by a wave of remagnetization [Figs. 9(c) and 9(d)]. Since at the first stage the magnetization near the edge was already reversed the process of remagnetization sweeps practically the whole particle. At the final stages when the tip passes the center the remagnetization reaches the opposite edge and the particle changes its direction of magnetization [Figs. 9(e) and 9(f)].

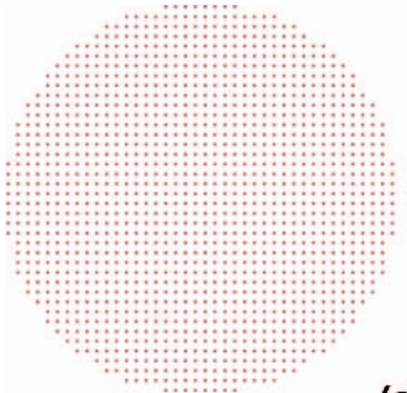

(a)
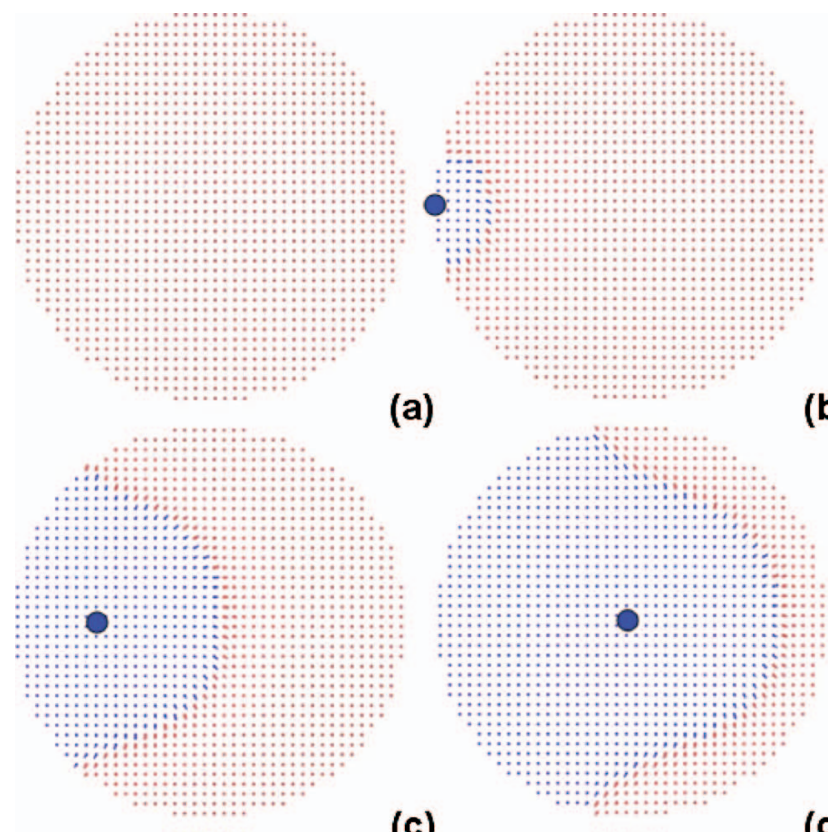

(b)
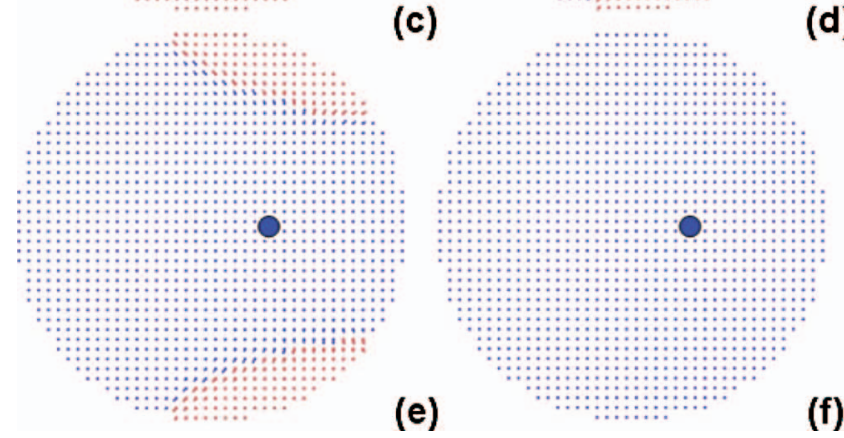

(d)

FIG. 9. (Color) Step-by-step stages of remagnetization by the MFM probe moving across the disk with $R_{d}>R_{t}\left(R_{d}=100 \mathrm{~nm}, R_{t}=50 \mathrm{~nm}\right)$. The probe position is indicated by the black circle. The positive $Z$-component of the magnetization is indicated by red and the negative $Z$-component by blue.

\section{B. MFM probe induced remagnetization of $35 \mathrm{~nm}$ CoPt nanodisks}

In the second series of experiments, we investigated the tip-induced remagnetization of $35 \mathrm{~nm}$ CoPt nanodisks with radius $R_{d}<R_{t}$. The sample was previously magnetized in a perpendicular external field of $15 \mathrm{kOe}$ in the opposite direction to the probe moment. The MFM constant height image of the initial state of $35 \mathrm{~nm}$ CoPt disks array is shown in Fig. 10.

The MFM tip-induced controllable writing on this particle array was also investigated experimentally. The writing to selected particles was achieved by reducing the tip-particle separation down to touching so a one-touch mechanism of remagnetization was realized. The step by step MFM images of the controllable bit writing on $35 \mathrm{~nm}$ CoPt nanodisks is demonstrated in Fig. 11.

The one-touch mechanism of remagnetization for $35 \mathrm{~nm}$ CoPt nanodisks was confirmed by LLG computer simulations. The step by step LLG model process of particle remagnetization by the inhomogeneous MFM probe magnetic field is shown in Fig. 12. The spherical probe $\left(R_{t}=30 \mathrm{~nm}\right)$ was situated over the center of particle and the tip-sample sepa- 


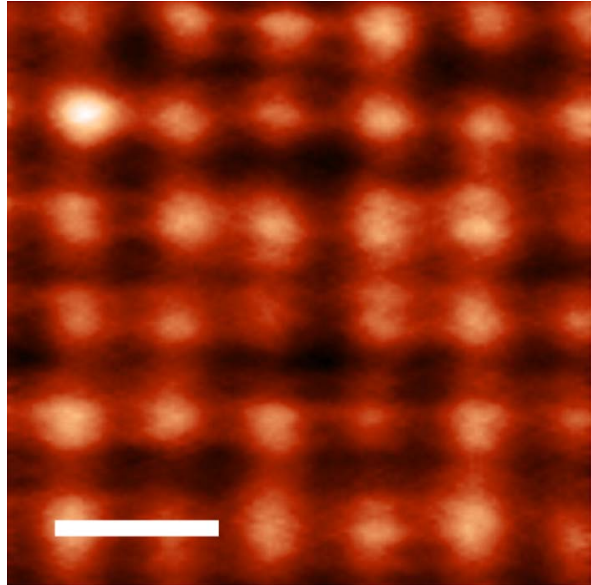

FIG. 10. (Color online) MFM image of perpendicularly magnetized $35 \mathrm{~nm}$ CoPt nanodisks. (The white scale bar is $200 \mathrm{~nm}$.)

ration was reduced down to touching. When the tip magnetic field exceeded some critical value the remagnetization was observed.

It is clearly seen in Fig. 12 that the remagnetization process goes through the inhomogeneous vortexlike state. The initial magnetization is directed up along the $Z$ axis [Fig. 12(a)]. At the first stage of remagnetization the radial component of magnetization appears (due to the radial component of the probe field, see Fig. 3) and afterwards the curling state is formed [Fig. 12(b)]. We think that the formation of this vortexlike state with a spiral distribution of magnetization is the result of competition between the magnetostatic energy through the particle edge and the Zeeman energy connected with the radial MFM tip magnetic field. At the next stage the magnetization in the narrow circular region changes the sign of the $Z$ component [blue region in Fig. $12(\mathrm{c})]$. Finally this nucleus with opposite $Z$ component spreads throughout the particle [Figs. 12(d)-12(f)]. The model remagnetization process calculated for a temperature of $300 \mathrm{~K}$ demonstrates a similar behavior of the magnetization but with a chaotic component caused by the influence of temperature.
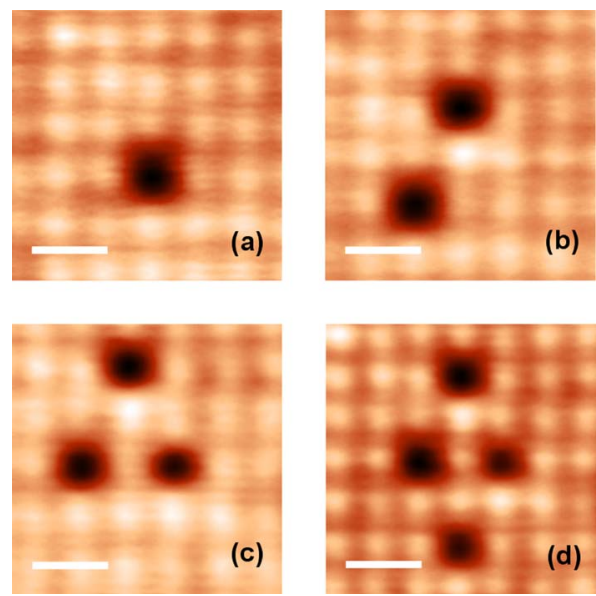

FIG. 11. (Color online) The MFM tip-induced bit writing in the ordered array of $35 \mathrm{~nm}$ CoPt nanoparticles. The remagnetization is accompanied by MFM contrast inversion. (a) is the MFM image with 1 reversed particle. (b) is for two reversed particles, (c) is for three reversed particles, and (d) is for four reversed particles. (The white scale bars are $200 \mathrm{~nm}$.)
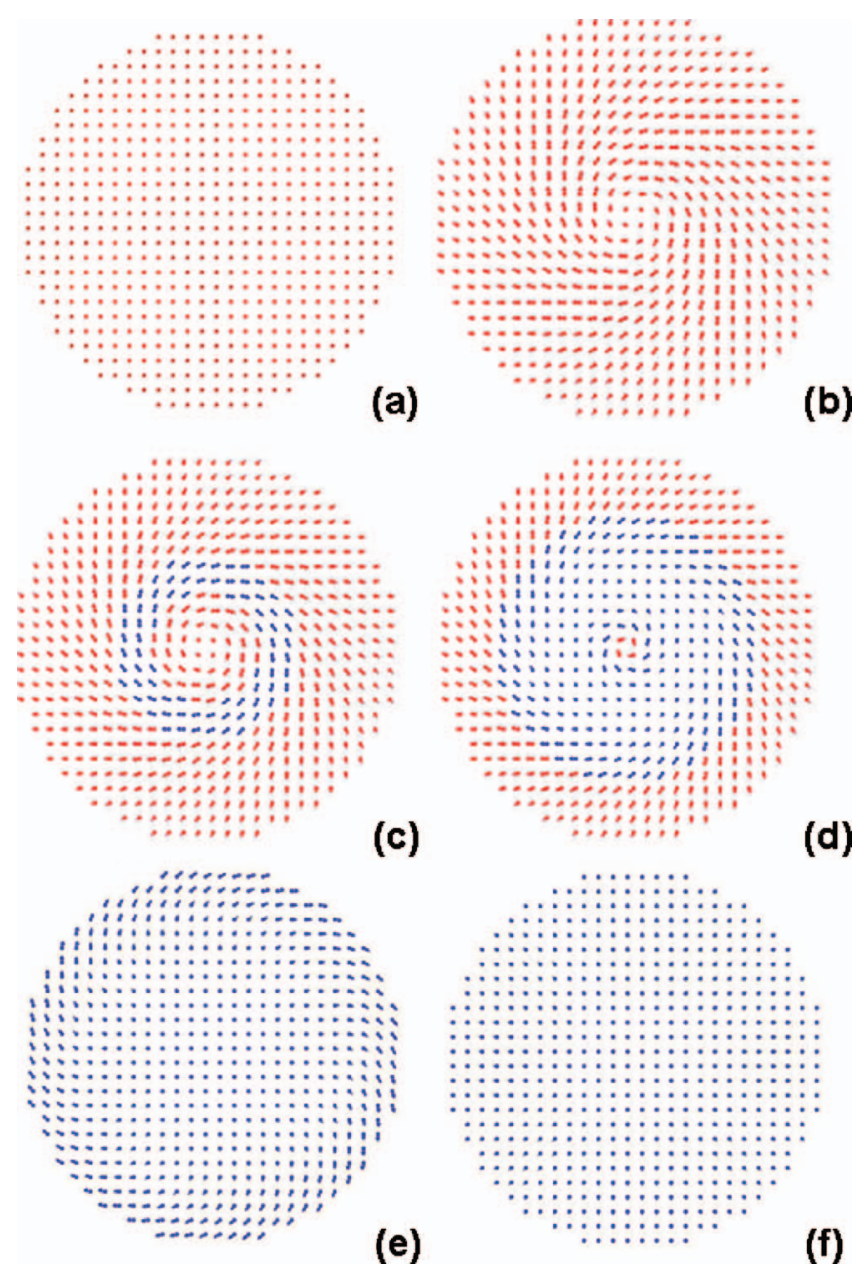

FIG. 12. (Color) Step-by-step stages of $35 \mathrm{~nm}$ CoPt nanodisk remagnetization by the MFM probe magnetic field (at a temperature of $0 \mathrm{~K}$ ). The probe was situated over the center of the particle and tip-sample separation was reduced down to touching. (a)-(e) are magnetization distributions at different times of the remagnetization process. The positive $Z$-component of the magnetization is indicated by red and the negative $Z$-component by blue.

As well as calculating the dynamics of magnetization in numerical simulations, we also determined the average disk's total energy to compare the energy barriers for remagnetization in a homogeneous external field $\Delta E_{\text {hom }}$ and for remagnetization in the nonhomogeneous field of an MFM tip $\Delta E_{\text {tip. }}$

The LLG simulations showed that remagnetization of 35 $\mathrm{nm}$ CoPt nanodisk in a homogeneous external field occurs by coherent rotation. In calculations we increased the magnitude of the external magnetic field right up to the start of remagnetization and the critical field parameters and value of total energy in the critical state $E_{\text {hom }}^{*}$ were determined. The value of the remagnetization barrier was calculated as the difference between the critical total energy $E_{\text {hom }}^{*}$ and the energy of the initial state $E_{0}$.

$$
\Delta E_{\mathrm{hom}}=E_{\mathrm{hom}}^{*}-E_{0} .
$$

On the other hand we calculated the effective energy barrier for non-homogeneous remagnetization of $35 \mathrm{~nm} \mathrm{CoPt}$ nanodisk in the nonhomogeneous field of a spherical MFM tip with an effective radius of $30 \mathrm{~nm}$. In these model calculations the probe radius $R_{t}$ was fixed and the magnitude of the tip moment was increased only by increasing the $M_{t}$ 
parameter, so the geometry of this numerical experiment was not changed. At the beginning of remagnetization, the critical field parameters and value of total energy in the critical state $E_{\text {tip }}^{*}$ were also determined. Thus the remagnetization barrier in the MFM tip field was calculated as

$$
\Delta E_{\text {tip }}=E_{\text {tip }}^{*}-E_{0} \text {. }
$$

The estimates based on LLG computer simulations showed first that the averaged $Z$ component of the critical field of the MFM tip is smaller than the value of the critical homogeneous field and second the energy barrier for tipinduced remagnetization is considerably lower than the barrier for magnetization reversal in a homogeneous magnetic field. For example, for $35 \mathrm{~nm}$ CoPt particle with an anisotropy constant $K=8 \times 10^{6} \mathrm{erg} / \mathrm{cm}^{3}$, the remagnetization barrier in the nonhomogeneous MFM tip field was $\Delta E_{\text {tip }}=5.7$ $\times 10^{-11}$ erg, while the barrier in an external homogeneous magnetic field was $\Delta E_{\mathrm{hom}}=11.7 \times 10^{-11} \mathrm{erg}$.

\section{CONCLUSION}

We reported the results of MFM investigations and micromagnetic model LLG calculations of MFM tip-induced remagnetization processes in $\mathrm{CoPt}$ nanodisks with a perpendicular magnetic anisotropy. We studied two different arrays of circular disks with a diameter of $200 \mathrm{~nm}\left(R_{d}>R_{t}\right)$ and 35 $\mathrm{nm}\left(R_{d}<R_{t}\right)$. The controllable MFM tip-induced bit writing on selected CoPt nanodisks was demonstrated in both cases, but writing processes had different mechanisms: the magnetization reversal of $200 \mathrm{~nm}$ disks was realized when the MFM probe moved across the particle, while in case of 35 $\mathrm{nm}$ nanodicsc one-touch remagnetization was obtained.

Computer simulations showed that in case of $200 \mathrm{~nm}$ disks the mechanism of embryo nucleation with reversed magnetization and further dynamic propagation following the probe moving across the particle was realized. On the other hand the one-touch MFM tip-induced remagnetization of $35 \mathrm{~nm}$ disks occurs through the inhomogeneous spiral vortexlike state.

In addition, the micromagnetic simulations showed that magnetization reversal in an inhomogeneous MFM probe field has a lower energy barrier in comparison with remagnetization of the same particles in a homogeneous external magnetic field, which occurs by a coherent rotation.

\section{ACKNOWLEDGMENTS}

The authors are very thankful to I. A. Nefedov, I. M. Shereshevsky I. A. Karetnikova, and N. I. Polushkin for assistance and fruitful discussions, to S.A. Michalski and R. D. Kirby for CoPt films fabrication, and to A. Yu. Klimov and V. V. Rogov for patterned samples preparation. Special thanks to reviewer for the very useful comments. This work was partly supported by the RFBR (Project No. 08-0201202) and by EC through the NANOSPIN project (Contract No. NMP4-CT-2004-013545).

${ }^{1}$ C. Chappert, H. Bernas, J. Ferré, V. Kottler, J.-P. Jamet, Y. Chen, E. Cambril, T. Devolder, F. Rousseaux, V. Mathet, and H. Launois, Science 280, 1919 (1998).
${ }^{2}$ M. Albrecht, A. Moser, C. T. Rettner, S. Anders, T. Thomson, and B. D. Terris, Appl. Phys. Lett. 80, 3409 (2002).

${ }^{3}$ J. I. Martin, J. Nogues, K. Liu, J. L. Vicent, and I. K. Schuller, J. Magn. Magn. Mater. 256, 449 (2003).

${ }^{4}$ M. H. Kryder and R. W. Gustafson, J. Magn. Magn. Mater. 287, 449 (2005)

${ }^{5}$ H. J. Richter, A. Y. Dobin, O. Heinonen, K. Z. Gao, R. J. M. d. Veerdonk, R. T. Lynch, J. Xue, D. Weller, P. Asselin, M. F. Erden, and R. M. Brockie, IEEE Trans. Magn. 42, 2255 (2006)

${ }^{6}$ A. Moser, O. Hellwig, D. Kercher, and E. Dobisz, Appl. Phys. Lett. 91, 162502 (2007).

${ }^{7}$ M. Albrecht, C. T. Rettner, A. Moser, M. E. Best, and B. D. Terris, Appl. Phys. Lett. 81, 2875 (2002).

${ }^{8}$ M. Albrecht, S. Anders, T. Thomson, C. T. Rettner, M. E. Best, A. Moser, and B. D. Terris, J. Appl. Phys. 91, 6845 (2002).

${ }^{9}$ K. Mitsuzuka, T. Shimatsu, H. Muraoka, H. Aoi, N. Kikuchi, and O. Kitakami, J. Appl. Phys. 103, 07C504 (2008).

${ }^{10}$ E. Chunsheng, V. Parekh, P. Ruchhoeft, S. Khizroev, and D. Litvinov, J. Appl. Phys. 103, 063904 (2008).

${ }^{11}$ V. Repain, J.-P. Jamet, N. Vernier, M. Bauer, J. Ferré, C. Chappert, J. Gierak, and D. Mailly, J. Appl. Phys. 95, 2614 (2004).

${ }^{12}$ M. V. Rastei, R. Meckenstock, and J. P. Bucher, Appl. Phys. Lett. 87, 222505 (2005)

${ }^{13}$ H.-J. Jang, P. Eames, E. Dan Dahlberg, M. Farhoud, and C. A. Ross, Appl. Phys. Lett. 86, 023102 (2005).

${ }^{14}$ S. Gider, J. Shi, D. D. Awschalom, P. F. Hopkins, K. L. Campman, A. C. Gossard, A. D. Kent, and S. von Molnár, Appl. Phys. Lett. 69, 3269 (1996).

${ }^{15}$ J. Lohau, A. Carl, S. Kirsch, and E. F. Wassermann, Appl. Phys. Lett. 78, 2020 (2001).

${ }^{16}$ H. Takahoshi, H. Saito, and S. Ishio, J. Magn. Magn. Mater. 272-276, E1313 (2004).

${ }^{17}$ M. Kleiber, F. Kümmerlen, M. Löhndorf, A. Wadas, D. Weiss, and R. Wiesendanger, Phys. Rev. B 58, 5563 (1998).

${ }^{18}$ X. Zhu, P. Grütter, V. Metlushko, and B. Ilic, Phys. Rev. B 66, 024423 (2002).

${ }^{19}$ X. Zhu, P. Grütter, V. Metlushko, and B. Ilic, J. Appl. Phys. 91, 7340 (2002).

${ }^{20}$ J. Chang, V. L. Mironov, B. A. Gribkov, A. A. Fraerman, S. A. Gusev, and S. N. Vdovichev, J. Appl. Phys. 100, 104304 (2006).

${ }^{21}$ V. L. Mironov, B. A. Gribkov, A. A. Fraerman, S. A. Gusev, S. N. Vdovichev, I. R. Karetnikova, I. M. Nefedov, and I. A. Shereshevsky, J. Magn. Magn. Mater. 312, 153 (2007).

${ }^{22}$ J. Chang, H. Yi, H. C. Koo, V. L. Mironov, B. A. Gribkov, A. A. Fraerman, S. A. Gusev, and S. N. Vdovichev, J. Magn. Magn. Mater. 309, 272 (2007).

${ }^{23}$ J. X. Shen, R. D. Kirby, K. Wierman, Z. S. Shan, D. J. Sellmyer, and T. Suzuki, J. Appl. Phys. 73, 6418 (1993).

${ }^{24}$ A. Aktag, S. Michalski, L. Yue, R. D. Kirby, and S.-H. Liou, J. Appl. Phys. 99, 093901 (2006)

${ }^{25}$ A. A. Fraerman, S. A. Gusev, L. A. Mazo, I. M. Nefedov, Yu. N. Nozdrin, I. R. Karetnikova, M. V. Sapozhnikov, I. A. Shereshevsky, and L. V. Sukchodoev, Phys. Rev. B 65, 064424 (2002).

${ }^{26}$ E. D. Boerner and H. N. Bertran, IEEE Trans. Magn. 33, 3052 (1997).

${ }^{27}$ G. Varvaro, E. Agostinelli, S. Laureti, A. M. Testa, J. M. Garcia-Martin, F. Briones, and D. Fiorani, J. Phys. D 41, 134017 (2008).

${ }^{28}$ M. Yu, Y. Liu, and D. J. Sellmyer, J. Appl. Phys. 87, 6959 (2000).

${ }^{29}$ S. H. Liou, S. Huang, E. Klimek, R. D. Kirby, and Y. D. Yao, J. Appl. Phys. 85, 4334 (1999).

${ }^{30}$ A. I. Gapin, X. R. Ye, J. F. Aubuchon, L. H. Chen, Y. J. Tang, and S. Jina, J. Appl. Phys. 99, 08G902 (2006).

${ }^{31}$ J. Goldstein, D. Newbury, P. Echlin, D. Joy, C. Fiori, and E. Lifshin, Scanning Electron Microscopy and X-ray Microanalysis (Plenum, New York, 1981).

${ }^{32}$ E. C. Stoner and E. P. Wohlfarth, Philos. Trans. R. Soc. Lond. A 240, 599 (1948).

${ }^{33}$ S. Okamoto, T. Kato, N. Kikuchi, O. Kitakami, N. Tezuka, and S. Sugimoto, J. Appl. Phys. 103, 07C501 (2008). 
${ }^{34}$ G. Hu, T. Thomson, C. T. Rettner, S. Raoux, and B. D. Terris, J. Appl. Phys. 97, $10 \mathrm{~J} 702$ (2005).

${ }^{35}$ N. Kikuchi, S. Okamoto, O. Kitakami, Y. Shimada, and K. Fukamichi, Appl. Phys. Lett. 82, 4313 (2003).
${ }^{36}$ K. Mitsuzuka, N. Kikuchi, T. Shimatsu, O. Kitakami, H. Aoi, H. Muraoka, and J. C. Lodder, IEEE Trans. Magn. 43, 2160 (2007).

${ }^{37}$ C. Moutafis, S. Komineas, C. A. F. Vaz, J. A. C. Bland, T. Shima, T. Seki, and K. Takanashi, Phys. Rev. B 76, 104426 (2007). 